\title{
Chiral strings, the sectorized description and their integrated vertex operators
}

\section{Renann Lipinski Jusinskas}

Institute of Physics of the Czech Academy of Sciences, CEICO - Central European Institute for Cosmology and Fundamental Physics, Na Slovance 2, 182 21, Prague, Czech Republic

E-mail: renannlj@fzu.cz

AbStract: A chiral string can be seen as an ordinary string in a singular gauge for the worldsheet metric and has the ambitwistor string as its tensionless limit. As proposed by Siegel, there is a one-parameter $(\beta)$ gauge family interpolating between the chiral limit and the usual conformal gauge in string theory. This idea was used to compute scattering amplitudes of tensile chiral strings, which are given by standard string amplitudes with modified ( $\beta$-dependent) antiholomorphic propagators.

Due to the absence of a sensible definition of the integrated vertex operator, there is still no ordinary prescription for higher than 3-point amplitude computations directly from the chiral model. The exception is the tensionless limit.

In this work this gap will be filled. Starting with a chiral string action, the integrated vertex operator is defined, relying on the so-called sectorized interpretation. As it turns out, this construction effectively emulates a left/right factorization of the scattering amplitude and introduces a relative sign flip in the propagator for the sector-split target space coordinates. $N$-point tree-level amplitudes can be easily shown to coincide with the results of Siegel et al. .

KeYwords: Conformal Field Models in String Theory, BRST Quantization, Scattering Amplitudes

ARXIV EPRINT: 1909.04069 


\section{Contents}

1 Introduction 1

2 Review of chiral strings 5

2.1 The Polyakov action and Siegel's gauge family 5

2.2 The sectorized interpretation 6

3 Vertex operators and amplitudes $\quad 8$

3.1 The sector-splitting operator $\quad 9$

$\begin{array}{lll}3.2 & \text { Integrated vertex operator and tree level amplitudes } & 11\end{array}$

$\begin{array}{lll}4 & \text { Final remarks } & 14\end{array}$

$\begin{array}{ll}\text { A A concrete example: the bosonic chiral string } & \mathbf{1 5}\end{array}$

$\begin{array}{ll}\text { A.1 Cohomology } & 15\end{array}$

$\begin{array}{ll}\text { A.2 3-point amplitudes at tree level } & 17\end{array}$

$\begin{array}{lll}\text { A.3 4-point amplitude with massless external states } & 19\end{array}$

\section{Introduction}

The ambitwistor string was proposed in [1] as a chiral string model underpinning the socalled CHY formulæ [2]. In spite of possessing an involved geometrical interpretation, the model has a simple realization. In the bosonic case, for instance, its chiral action takes the gauge fixed form

$$
S_{\mathrm{bos}}=\frac{1}{2 \pi} \int d^{2} z\left\{P_{m} \bar{\partial} X^{m}+b \bar{\partial} c+\tilde{b} \bar{\partial} \tilde{c}\right\},
$$

where $X^{m}$ denote the target space coordinates, $c$ is the holomorphic component of reparametrization ghost and $\tilde{c}$ is the ghost associated to the massless constraint $H \equiv P^{m} P_{m}=0$. Incidentally, this action resembled the one proposed earlier by Hohm, Siegel and Zwiebach (HSZ) [3], which was considered in the context of doubled-coordinate field theory towards a model with manifest T-duality. The ambitwistor string, however, has no dimensionful parameter.

The connection between the ambitwistor string and the ordinary string was not precisely understood at first. Due to the massless constraint and its finite spectrum, it might be more intuitive to picture the ambitwistor string as arising from some infinite tension (zero length) limit of the string. In this case, the string shrinks to a point and massive states become infinitely heavy, decoupling from the theory. In terms of the action, this idea is supported classically when the infinite tension limit is accompanied by singular field redefinitions $[1,4]$. 
On the other hand, the infinite length limit of the string yields too a massless spectrum. The difference is that it consists of an infinite number of higher spin states. From this point of view, if the ambitwistor string is supposed to come from a zero tension limit, it has to be concomitant with some process that truncates the physical spectrum. In [5], Siegel proposed a one-parameter family of gauges that interpolates between the usual conformal gauge and the chiral gauge leading to the action above. In terms of the metric $g_{i j}$, where $\{i, j\}=\tau, \sigma$ are the standard worldsheet directions, it corresponds to

$$
g_{i j}=e^{2 \omega}\left(\begin{array}{cc}
-1+\beta & \beta \\
\beta & 1+\beta
\end{array}\right),
$$

where $\omega$ is a conformal factor and $\beta \geq 0$ is the parameter. The conformal gauge is recovered when $\beta=0$, while the chiral limit is obtained when $\beta \rightarrow \infty$. The action (1.1) is the outcome of the BRST quantization of the first order form of the Polyakov action in this gauge (referred to as HSZ). At least at the classical level, the ambitwistor string can then be seen as the tensionless limit of the usual string in the singular HSZ gauge. ${ }^{1}$ This idea seems to be directly connected with the results of Gamboa et al. [6, 7], where different ordering prescriptions lead to different spectra in the tensionless limit of the (spinning) string, called null string. Later, in [8], this connection was made much more clear and precise.

In addition to the bosonic model, the extensions of the ambitwistor string with $\mathcal{N}=1$ (heterotic) and $\mathcal{N}=2$ (types IIA and IIB) worldsheet supersymmetry were also analyzed in [1]. Their descriptions with manifest spacetime supersymmetry appeared soon after in [9], using the pure spinor formalism. If compact spacetime dimensions are analyzed, naturally introducing a dimensionful parameter, the physical spectrum is much richer and an infinite number of higher spin massive states are present [10].

For the type II case, the ambitwistor spectrum coincides with the usual massless superstring spectrum $(\mathcal{N}=2, d=10$ supergravity) and, again, the infinite tension picture seems adequate. The same agreement is observed for the gauge sector of the heterotic model. On the other hand, both the bosonic model and the gravity sector of the heterotic model do not match their usual string counterparts. In fact, a more thorough analysis of their BRST cohomology shows that the physical spectrum is non-unitary. In particular, the kinetic action associated to these states involves higher derivatives. This was only recently demonstrated [11].

This asymmetry between the gravity sector of the bosonic, heterotic and type II ambitwistor spectrum is more easily understood when the tensile picture is considered. In this case, the massless ambitwistor constraint is replaced by

$$
P_{m} P^{m}+\mathcal{T}^{2} \partial X_{m} \partial X^{m}=0
$$

where $\mathcal{T}$ is the string tension. In principle, this tensile "deformation" allows for massive states in the spectrum. However, this depends on the amount of supersymmetry of the

\footnotetext{
${ }^{1}$ Singular because, strictly speaking, the limit $\beta \rightarrow \infty$ is not a valid gauge choice since it cannot be inverted. Any finite $\beta$, however, can be put back in the conformal gauge through an extra coordinate transformation.
} 
model. For $\mathcal{N}=2$, the physical spectrum of the chiral string is independent of the tension. For $\mathcal{N}=1$ (heterotic), the physical spectrum contains the usual supergravity states plus a multiplet resembling the first massive level of the open superstring with mass $m^{2}=4 \mathcal{T}$. For $\mathcal{N}=0$ (bosonic), in addition to the usual gravity states (graviton, dilaton and KalbRamond form), the spectrum contains two spin 2 fields with mass $m^{2}= \pm 4 \mathcal{T}$.

As proposed in [5], the tensile chiral string can be obtained from a singular gauge limit of the usual string together with a change in the worldsheet boundary conditions, which effectively amounts to a sign flip in the antiholomorphic part of the $\langle X X\rangle$ propagator. This idea was explored by Huang et al. in [12], where a modified Kawai-Lewellen-Tye factorization was used to compute tree level amplitudes. As expected, the poles of these amplitudes coincide with the physical spectrum described above. In the tensionless limit, the massive spin 2 fields of the bosonic and the heterotic model become massless and mix with the graviton. They can be understood as auxiliary fields responsible for introducing the higher derivative equations of motion for the original massless states. This construction has been demonstrated in the bosonic model in [13], including current algebra extensions.

The first loop computations using the ambitwistor string appeared in [14], indicating the one-loop modular invariance of the GSO projected type II scattering amplitudes. Results on two-loops appeared more recently in [15]. It seems, however, that the purely bosonic model does not share such feature. This was analyzed within the framework of null string theory in [16] and the results agree with the tensile model of [17]. Unfortunately, modular invariance cannot yet be confirmed using the pure spinor construction of [9]. The reason is that the $b$ ghost in the pure spinor formalism is a composite operator and its definition in the tensionless limit has not been found so far. In the tensile model, however, the $b$ ghost was successfully built in [18], which introduced the sectorized description of the chiral models. The idea behind this interpretation is that a chiral model with action of the form (1.1) can be effectively described in terms of two sectors that emulate the usual left and right movers of the (super) string.

Since the pure spinor formalism still lacked a fundamental worldsheet description, the relation between the chiral actions of [18] and [5] was not very clear. This was only completely achieved later in [19], where the equivalent of the HSZ gauge was implemented with the derivation of the chiral action of [18]. Another issue that prevented a more immediate identification of the sectorized model with Siegel's chiral string was the absence of the integrated vertex operator (IVO) in the former. Therefore, only 3-point tree-level amplitudes could be directly compared.

In general, chiral models lack a natural ingredient to define an IVO as an integration over a Riemann surface, since all fields are holomorphic. In the ambitwistor string, this obstacle is overcome with the insertion of the BRST-closed operators $\bar{\delta}\left(k^{m} P_{m}\right)$, where $k^{m}$ is the linear momentum of the vertex. In turn, this operator enforces the localization of the scattering amplitudes. The definition of the operator $\bar{\delta}\left(k^{m} P_{m}\right)$ was made more precise by Ohmori in [20] and can be written instead as $\bar{\delta}\left(H_{-1}\right)$, where $H_{-1}$ is the mode -1 of $H$ acting on the unintegrated vertex operator. It coincides with $\bar{\delta}\left(k^{m} P_{m}\right)$ when the $X^{m}$ dependence of the vertex operator is only through $e^{i k \cdot X}$. 
In this work, Ohmori's construction will be generalized in order to properly define the IVO of the tensile chiral string. The main ingredient in this construction is the sectorsplitting operator $\bar{\Delta}$, formally defined as

$$
\bar{\Delta} \equiv e^{i \bar{z} \mathcal{H}_{-1}},
$$

where $\mathcal{H}_{-1}$ is the -1 mode of the BRST-exact generalization of the left-hand side of equation (1.3). This operator has a simple interpretation in the sectorized description, generating a point-splitting between operators of different sectors. In particular, its action on the target space coordinate $X^{m}$ generates a scalar field of the form $\bar{X}^{m}\left(z^{+}, z^{-}\right) \equiv$ $\bar{\Delta} \cdot X^{m}(z)$, where the coordinates $z^{ \pm}=z \pm i \mathcal{T} \bar{z}$ can be interpreted as conjugate to each other. ${ }^{2}$ It satisfies the OPE

$$
\bar{X}^{m}\left(z^{+}, z^{-}\right) \bar{X}^{n}(0,0) \sim \frac{\eta^{m n}}{2 \mathcal{T}} \ln \left(\frac{z^{-}}{z^{+}}\right),
$$

emulating the boundary condition change proposed by Siegel in [5]. Following this result, a series of identifications is put forward, resulting in a simple map between the chiral models with first order action of the form (1.1) and ordinary second order string actions in the conformal gauge but with the particular OPE above. It is then straightforward to show that tree level scattering amplitudes in the sectorized model are given by the "flipped" Kawai-Lewellen-Tye factorized amplitudes of [12]. In order to see the tensionless limit, it is enough to show that

$$
\lim _{\mathcal{T} \rightarrow 0} \bar{\Delta} \cdot X^{m}(z)=X^{m}(z)+i \bar{z} P^{m}(z)
$$

and the modular integration of $\bar{z}$ in the IVO's generates the localization operators $\bar{\delta}\left(k^{m} P_{m}\right)$.

It is worth to point out that a rigorous way of defining $N$-point amplitudes in these chiral models already exists and it is given in terms of intersection numbers of cohomology classes on the moduli space of punctured Riemann surfaces (see [21] and references therein). Their equivalence with the amplitudes of [12] was already demonstrated in [22] using intersection theory. ${ }^{3}$ On the other hand, the definition of the integrated vertex operator for the chiral models brings this equivalence closer to the usual construction in string theory, with a more physical approach.

This work is organized as follows. Section 2 consists of a review of Siegel's gauge family of [5] and the sectorized interpretation in the chiral limit. Section 3 introduces the operator (1.4) and its properties, finally leading to the definition of the integrated vertex operator and the computation of $N$-point tree level scattering amplitudes directly from the chiral model. Section 4 presents some quick final remarks and possible directions to follow. In the appendix, the bosonic chiral string is analyzed as an example. The 4-point amplitude with external massless states is computed and some properties are verified, e.g. Möbius invariance and the existence of physical poles only at $m^{2}=0$ and $m^{2}= \pm 4 \mathcal{T}$.

\footnotetext{
${ }^{2}$ As long as the moduli space of $z$ and $\bar{z}$ corresponds to the complex plane, $\bar{z}$ does not necessarily have to be the complex conjugate of $z$. This notation was chosen for the sake of simplicity. The reader may find useful the discussion in [20] regarding the analysis of the moduli space of chiral strings.

${ }^{3}$ I would like to thank Sebastian Mizera for bringing this to my attention.
} 


\section{Review of chiral strings}

In this section, the singular gauge fixing leading to chiral strings will be reviewed in the context of Siegel's gauge family and the sectorized description.

\subsection{The Polyakov action and Siegel's gauge family}

The Polyakov action in the Hamiltonian form is given by

$$
\begin{gathered}
S_{P}=\frac{1}{2 \pi} \int d \tau d \sigma\left\{P_{m} \partial_{\tau} X^{m}+\frac{1}{4 \mathcal{T}} e_{+}\left(P_{m}+\mathcal{T} \partial_{\sigma} X_{m}\right)\left(P^{m}+\mathcal{T} \partial_{\sigma} X^{m}\right)\right. \\
\left.+\frac{1}{4 \mathcal{T}} e_{-}\left(P_{m}-\mathcal{T} \partial_{\sigma} X_{m}\right)\left(P^{m}-\mathcal{T} \partial_{\sigma} X^{m}\right)\right\},
\end{gathered}
$$

where $\mathcal{T}$ is the string tension and $e_{ \pm}$denote Weyl invariant Lagrange multipliers. They can be effectively interpreted as the gauge fields of worldsheet reparametrization symmetry, with generators

$$
H_{ \pm} \equiv\left(P_{m} \pm \mathcal{T} \partial_{\sigma} X_{m}\right)\left(P^{m} \pm \mathcal{T} \partial_{\sigma} X^{m}\right)
$$

The associated gauge transformations, with local parameters $c_{+}$and $c_{-}$, are given by

$$
\begin{aligned}
\delta X^{m} & =\frac{1}{2}\left[c_{+}\left(P^{m}+\mathcal{T} \partial_{\sigma} X^{m}\right)+c_{-}\left(P^{m}-\mathcal{T} \partial_{\sigma} X^{m}\right)\right], \\
\delta P_{m} & =\frac{\mathcal{T}}{2} \partial_{\sigma}\left[c_{+}\left(P^{m}+\mathcal{T} \partial_{\sigma} X^{m}\right)-c_{-}\left(P^{m}-\mathcal{T} \partial_{\sigma} X^{m}\right)\right], \\
\delta e_{ \pm} & =\partial_{\tau} c_{ \pm} \pm\left(c_{ \pm} \partial_{\sigma} e_{ \pm}-e_{ \pm} \partial_{\sigma} c_{ \pm}\right),
\end{aligned}
$$

and the usual conformal gauge is obtained by setting $e_{ \pm}=-1$.

In [5], Siegel introduced a one-parameter $(\beta)$ family of gauges expressed as

$$
e_{ \pm}=\frac{ \pm \beta-1}{\beta+1} .
$$

This gauge family can be seen as an interpolation between the conformal gauge $(\beta=0)$ and the chiral gauge $(\beta \rightarrow \infty)$, which was first proposed in the doubled-coordinate field theory context of [3].

The chiral gauge is singular and has a simple geometric interpretation. First recall that $e_{+}$and $e_{-}$can be expressed in terms of the worldsheet metric $g_{i j}\left(g^{i j}\right)$ as

$$
e_{ \pm} \equiv \pm \frac{g^{\tau \sigma}}{g^{\tau \tau}}-\frac{1}{g^{\tau \tau} \sqrt{-g}}
$$

Given the gauge choice (2.4), it follows that

$$
g_{i j}=\left(\begin{array}{cc}
-1+\beta & \beta \\
\beta & 1+\beta
\end{array}\right),
$$

while $\operatorname{det} g_{i j}=-1$. In principle, there is also a conformal factor but it can be conveniently tuned for a given topology using the Weyl symmetry (for simplicity, only the flat worldsheet will be discussed here). The line element can be written in terms of the light-cone 


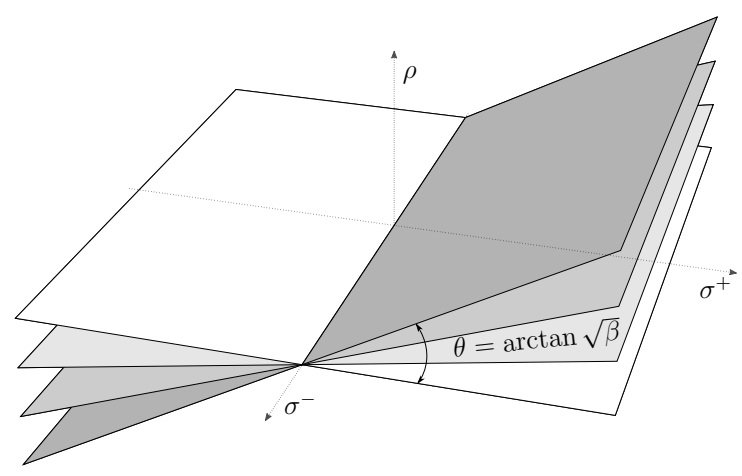

Figure 1. Increasing $\beta$ corresponds to increasing the angle $\theta$ of the metric plane with respect to the $\sigma^{+} \sigma^{-}(\beta=0)$ plane. The $\beta \rightarrow \infty$ limit corresponds to the $\sigma^{+}=0$ plane.

coordinates, $\sigma^{ \pm}=\tau \pm \sigma$, as $d s^{2}=-d \sigma^{+} d \sigma^{-}+\beta\left(d \sigma^{+}\right)^{2}$. In order to picture this, it is useful to consider a three-dimensional flat space with line element $d s^{2}=-d \sigma^{+} d \sigma^{-}+d \rho^{2}$ subjected to the constraint $\rho=\sqrt{\beta} \sigma^{+}$. The intrinsic two-dimensional metric is then given by equation (2.6) and corresponds to a plane inclined by an angle $\theta=\arctan \sqrt{\beta}$ with respect to $\rho=0$. See figure 1 .

Physically, increasing $\beta$ implies that the line element is dominated by $d \sigma^{+}$. In the limit $\beta \rightarrow \infty, d \sigma^{-}$becomes irrelevant and the worldsheet dynamics is solely governed by the coordinate $\sigma^{+}$. For Euclidean worldsheet coordinates, this limit leads to the (chiral) gauge fixed action

$$
S=\frac{1}{2 \pi} \int d^{2} z\left\{P_{m} \bar{\partial} X^{m}+b_{+} \bar{\partial} c_{+}+b_{-} \bar{\partial} c_{-}\right\}
$$

where the gauge parameters $c_{ \pm}$were promoted to anticommuting ghosts with antighosts $b_{ \pm}$. Note, in addition, that the string tension $\mathcal{T}$ does not explicitly appear.

\subsection{The sectorized interpretation}

The action (2.7) can be effectively interpreted in terms of two sectors "+" and "-", analogous to the usual left and right-movers of the bosonic string. This will become clearer in the next section. To each sector, a characteristic energy-momentum tensor can be assigned, given by

$$
\begin{aligned}
& T_{+}=-\frac{1}{4 \mathcal{T}} P_{m}^{+} P_{n}^{+} \eta^{m n}-2 b_{+} \partial c_{+}+c_{+} \partial b_{+}, \\
& T_{-}=\frac{1}{4 \mathcal{T}} P_{m}^{-} P_{n}^{-} \eta^{m n}-2 b_{-} \partial c_{-}+c_{-} \partial b_{-},
\end{aligned}
$$

where

$$
P_{m}^{ \pm} \equiv P_{m} \pm \mathcal{T} \partial X_{m}
$$

and $\eta^{m n}$ is the flat target-space metric. The BRST charge $Q$ manifestly expresses the sectorization as $Q=Q^{+}+Q^{-}$, such that

$$
Q^{ \pm} \equiv \oint\left\{c_{ \pm} T_{ \pm}-b_{ \pm} c_{ \pm} \partial c_{ \pm}\right\}
$$


As in the usual bosonic string, the number of spacetime dimensions is $d=26$, fixed by requiring the nilpotency of $Q$.

While the combination $\left(T_{+}+T_{-}\right)$gives the left-moving Virasoro generator, $\left(T_{+}-T_{-}\right)$ takes the form of a generalized particle-like Hamiltonian:

$$
\begin{aligned}
T & =T_{+}+T_{-} \\
& =-P_{m} \partial X^{m}-b \partial c-\partial(b c)-\tilde{b} \partial \tilde{c}-\partial(\tilde{b} \tilde{c}) \\
\mathcal{H} & \equiv \mathcal{T}\left(T_{+}-T_{-}\right) \\
& =-\frac{1}{2} P_{m} P^{m}-\frac{\mathcal{T}^{2}}{2} \partial X_{m} \partial X^{m}-\mathcal{T}\left(b_{+} \partial c_{+}-b_{-} \partial c_{-}\right)+\mathcal{T} \partial\left(c_{+} b_{+}-c_{-} b_{-}\right) .
\end{aligned}
$$

Since $\left\{Q, b_{ \pm}\right\}=T_{ \pm}$, both $T$ and $\mathcal{H}$ are BRST-exact. Note that $T_{+}$and $T_{-}$may have the interpretation of energy-momentum tensors only within their respective sector.

The BRST charge can be cast in a more traditional form as

$$
Q=\oint\left\{c T-b c \partial c+\frac{1}{2} \tilde{c} \mathcal{H}+\ldots\right\}
$$

where the ... denotes terms of order $\mathcal{T}^{1}$ and $\mathcal{T}^{2}$, and

$$
\begin{aligned}
c \equiv \frac{1}{2}\left(c_{+}+c_{-}\right), & \tilde{c} \equiv \frac{1}{2 \mathcal{T}}\left(c_{-}-c_{+}\right), \\
b \equiv\left(b_{+}+b_{-}\right), & \tilde{b} \equiv \mathcal{T}\left(b_{-}-b_{+}\right) .
\end{aligned}
$$

$Q$ resembles the bosonic ambitwistor BRST charge proposed by Mason and Skinner [1] and, in fact, can be thought of as its tensile generalization.

The sectorized description can be extended fairly easily as long as the chiral action of the models under consideration have vanishing characteristic central charges, corresponding to the absence of quartic poles in the OPE's

$$
T_{ \pm}(z) T_{ \pm}(y) \sim \frac{2 T_{ \pm}}{(z-y)^{2}}+\frac{\partial T_{ \pm}}{(z-y)}
$$

where $T_{+}$and $T_{-}$can be generically written as

$$
T_{ \pm}=\mp \frac{1}{4 \mathcal{T}} P_{m}^{ \pm} P_{n}^{ \pm} \eta^{m n}+T_{ \pm}^{\text {ghost }}+T_{ \pm}^{\text {matter }}
$$

In other words, the central charge of the chiral model has to vanish and $\mathcal{H}$ has to be a primary operator.

Some examples of interest include the spinning string with $\mathcal{N}=(1,1), \mathcal{N}=(1,0)$ and $\mathcal{N}=(0,1)$ worldsheet supersymmetry, the pure spinor superstring with $\mathcal{N}=2$ and $\mathcal{N}=1$ spacetime supersymmetry ${ }^{4}$ or purely bosonic models in $d<26$ extended with current algebras. All these models contain graviton excitations (either type II, heterotic or bosonic), with or without ghosts, and recover the corresponding ambitwistor string in their tensionless limit.

\footnotetext{
${ }^{4}$ In the pure spinor formalism, $b_{+}$and/or $b_{-}$are composite operators satisfying $\left\{Q, b_{ \pm}\right\}=T_{ \pm}$.
} 


\section{$3 \quad$ Vertex operators and amplitudes}

In the chiral strings mentioned above, physical states are defined to be in their ghost number 2 BRST cohomology and annihilated by $b_{0}$, the zero mode of the $b$ ghost $c f$. equation (2.13). This last requirement is the chiral analogous of the condition $\left(b_{0}-\bar{b}_{0}\right)=0$ for off-shell closed string states and was shown to provide the correct prescription for ambitwistor strings [11] (see $[24,25]$ for more details on the definition of off-shell states and the ambitwistor string field theory).

The unintegrated vertex operators are straightforward to build (normal ordering is assumed whenever needed). For momentum eigenstates, their different components have the generic form

$$
U\left(z ; k^{m}\right)=U_{+}(z) U_{-}(z) e^{i k \cdot X(z)},
$$

where $U_{+}$and $U_{-}$are composite operators constructed exclusively from the "+" and "-" sectors respectively. As long as the physical state conditions are satisfied, $U_{+}$and $U_{-}$do not necessarily have the same ghost number.

The definition of integrated vertex operators is slightly more intricate. Because the model is chiral, the usual recipe from string theory does not work. Naively, the integrated vertex operator could be defined as

$$
V\left(z ; k^{m}\right) \stackrel{?}{\equiv}\left(b_{-}\right)_{-1} \cdot\left(b_{+}\right)_{-1} \cdot U\left(z ; k^{m}\right),
$$

where the subscript -1 denotes the corresponding mode of the $b$ ghost operators. Note, however, that $V$ has conformal weight $(2,0)$ and satisfies $\bar{\partial} V=0$, therefore the integration over the Riemann surface $\Sigma$ is not well defined. In addition,

$$
\begin{aligned}
{\left[Q, V\left(z ; k^{m}\right)\right] } & =\left(T_{-}\right)_{-1} \cdot\left(b_{+}\right)_{-1} \cdot U\left(z ; k^{m}\right)-\left(b_{-}\right)_{-1} \cdot\left(T_{+}\right)_{-1} \cdot U\left(z ; k^{m}\right) \\
& =\frac{1}{2} T_{-1} \cdot\left(b_{-}-b_{+}\right)_{-1} \cdot U\left(z ; k^{m}\right)+\frac{1}{2 \mathcal{T}} \mathcal{H}_{-1} \cdot\left(b_{+}+b_{-}\right)_{-1} \cdot U\left(z ; k^{m}\right) \\
& =\frac{1}{2} \frac{\partial}{\partial z}\left[\left(b_{-}-b_{+}\right)_{-1} \cdot U\left(z ; k^{m}\right)\right]+\frac{1}{2 \mathcal{T}} \mathcal{H}_{-1} \cdot\left(b_{+}+b_{-}\right)_{-1} \cdot U\left(z ; k^{m}\right) .
\end{aligned}
$$

While the first term on the right hand side of the last equation can be disregarded as a boundary contribution in the moduli space integration, the second term does not vanish and the integrated vertex operator is not BRST invariant.

A natural way of fixing these issues would be to formally introduce a BRST closed operator $\bar{\delta}\left(\mathcal{H}_{-1}\right)$ with conformal weight $(-1,1)$, such that the product $\bar{\delta}\left(\mathcal{H}_{-1}\right) \cdot \mathcal{H}_{-1}$ vanishes under the appropriate conditions, e.g. corresponding to a boundary of the moduli space. In this case, the integrated vertex operator should be defined as

$$
V\left(z ; k^{m}\right) \equiv\left(b_{-}\right)_{-1} \cdot\left(b_{+}\right)_{-1} \cdot \bar{\delta}\left(\mathcal{H}_{-1}\right) \cdot U\left(z ; k^{m}\right),
$$

which satisfies $\left[Q, V\left(z ; k^{m}\right)\right]=\frac{\partial}{\partial z}(\ldots)$. In the tensionless limit, this idea was proposed by Ohmori in [20] and agrees with the operator $\bar{\delta}(k \cdot P)$ introduced by Mason and Skinner in [1] as long as the only dependence of the vertex operator on the target-space coordinates is through $e^{i k \cdot X}$. The moduli space integration is then well defined and the role of $\bar{\delta}(k \cdot P)$ is to impose the so-called scattering equations in the $N$-point amplitude computations. 
In this section, the integrated vertex operator for the sectorized model will be finally built, motivated by the definition (3.4). Besides providing a more robust and intuitive definition of the operator $\bar{\delta}\left(\mathcal{H}_{-1}\right)$, the tools developed here explain the expected connection between the sectorized model and the $\beta \rightarrow \infty$ limit of Siegel's string [5].

\subsection{The sector-splitting operator}

Consider the BRST-closed operator

$$
\begin{aligned}
\bar{\Delta} & \equiv \sum_{n=0}^{\infty} \frac{1}{n !}\left(i \bar{z} \mathcal{H}_{-1}\right)^{n}, \\
& =e^{i \bar{z} \mathcal{H}_{-1}},
\end{aligned}
$$

where

$$
\mathcal{H}_{-1}=\mathcal{T} \oint\left\{T_{+}-T_{-}\right\}
$$

By construction,

$$
\begin{aligned}
{\left[L_{0}, \bar{\Delta}\right] } & =\bar{z} \bar{\partial} \bar{\Delta} \\
\bar{\Delta} \cdot \mathcal{H}_{-1} & =-i \bar{\partial} \bar{\Delta}
\end{aligned}
$$

where $L_{0}$ is the Virasoro zero mode.

The action of the operator $\bar{\Delta}$ can be understood in terms of the sectorized interpretation as follows. While $T=T_{+}+T_{-}$generates holomorphic worldsheet translations,

$$
e^{z L_{-1}} \mathcal{O}(0)=\mathcal{O}(z),
$$

there is no analogous operation for the antiholomorphic sector because the classical equations of motion imply $\bar{\partial}=0$. As discussed in subsection 2.1, this is a consequence of the singular metric gauge (HSZ). The antiholomorphic dependence is then artificially reintroduced by the operator $\bar{\Delta}$. This operation depends on the string tension $\mathcal{T}$ and on the sector on which the operator acts. Observe, for example, that

$$
\bar{\Delta} \cdot P_{m}^{ \pm}(z)=P_{m}^{ \pm}(z \pm i \mathcal{T} \bar{z})
$$

and, more generally,

$$
\bar{\Delta} \cdot \mathcal{O}^{ \pm}(z)=\mathcal{O}^{ \pm}(z \pm i \mathcal{T} \bar{z})
$$

where $\mathcal{O}^{+}\left(\mathcal{O}^{-}\right)$denotes operators exclusively built from the plus (minus) sector. Therefore, $\bar{\Delta}$ creates a point-splitting between the two sectors. The presence of the string tension in the arguments is unusual but makes it easier to determine the tensionless limit. It could have been removed, of course, by a redefinition of (3.6) as $\bar{\Delta}=\exp \left\{\frac{i}{\mathcal{T}} \bar{z} \mathcal{H}_{-1}\right\}$. It will be convenient to define also the sector coordinates $z^{ \pm}$as

$$
z^{ \pm} \equiv z \pm i \mathcal{T} \bar{z}, \partial_{ \pm} \equiv \frac{1}{2 \mathcal{T}}(\mathcal{T} \partial \mp i \bar{\partial})
$$

such that $\partial_{ \pm} z^{ \pm}=1$ and $\partial_{ \pm} z^{\mp}=0$. These coordinates play the role of the usual holomorphic and anti-holomorphic pair, but they are not suitable for the tensionless limit analysis since they become degenerate, i.e. $z^{ \pm} \rightarrow z$ for $\mathcal{T} \rightarrow 0$. 
The only non-trivial operation is the action of $\bar{\Delta}$ on the target-space coordinates, $X^{m}$, since they do not belong to a specific sector. It will be defined as

$$
\bar{X}_{m}(z, \bar{z}) \equiv \bar{\Delta} \cdot X_{m}(z)
$$

and it is straightforward to show from the series expansion (3.6) that

$$
\begin{aligned}
\bar{X}_{m}(z, \bar{z}) & =X_{m}(z)+\frac{1}{2} \sum_{n=1}^{\infty} \frac{i \bar{z}}{n !}(i \bar{z} \mathcal{T})^{n-1}\left\{\partial^{n-1} P_{m}^{+}(z)+(-1)^{n-1} \partial^{n-1} P_{m}^{-}(z)\right\} \\
& =\frac{1}{2} X_{m}(z+i \mathcal{T} \bar{z})+\frac{1}{2} X_{m}(z-i \mathcal{T} \bar{z})+i \bar{z} \sum_{n=0}^{\infty} \frac{(i \bar{z} \mathcal{T})^{2 n}}{(2 n+1) !} \partial^{2 n} P_{m}(z)
\end{aligned}
$$

To obtain the second line from the first line, it suffices to recall the definition (2.9). Note that $\bar{X}_{m}(z, \bar{z})$ satisfies the equations of motion

$$
\begin{aligned}
& \bar{\partial} \bar{X}_{m}(z, \bar{z})=\frac{1}{2} P_{m}^{+}(z+i \mathcal{T} \bar{z})+\frac{1}{2} P_{m}^{-}(z-i \mathcal{T} \bar{z}), \\
& \partial \bar{X}_{m}(z, \bar{z})=\frac{1}{2 \mathcal{T}} P_{m}^{+}(z+i \mathcal{T} \bar{z})-\frac{1}{2 \mathcal{T}} P_{m}^{-}(z-i \mathcal{T} \bar{z}),
\end{aligned}
$$

which can be rewritten in a more suggestive form in terms of the coordinates $z^{ \pm}$

$$
\begin{aligned}
\partial_{+} \bar{X}_{m} & =\frac{1}{2 \mathcal{T}} P_{m}^{+}\left(z^{+}\right), \\
\partial_{-} \bar{X}_{m} & =-\frac{1}{2 \mathcal{T}} P_{m}^{-}\left(z^{-}\right), \\
\partial_{+} \partial_{-} \bar{X}_{m} & =0 .
\end{aligned}
$$

The BRST transformation of $\bar{X}^{m}$ also incorporates such sector splitting as

$$
\begin{aligned}
{\left[Q, \bar{X}_{m}(z, \bar{z})\right] } & =\frac{1}{2 \mathcal{T}} c_{+} P_{m}^{+}(z+\bar{z} \mathcal{T})-\frac{1}{2 \mathcal{T}} c_{-} P_{m}^{-}(z-\bar{z} \mathcal{T}) \\
& =c_{+} \partial_{+} \bar{X}_{m}\left(z^{+}\right)+c_{-} \partial_{-} \bar{X}_{m}\left(z^{-}\right)
\end{aligned}
$$

All these results suggest that $\bar{X}^{m}$ behaves like an ordinary worldsheet scalar from a second order action, including a non-trivial OPE with itself which can be computed to be

$$
\bar{X}^{m}\left(z^{+}, z^{-}\right) \bar{X}^{n}\left(y^{+}, y^{-}\right) \sim-\frac{\eta^{m n}}{2 \mathcal{T}} \ln \left(z^{+}-y^{+}\right)+\frac{\eta^{m n}}{2 \mathcal{T}} \ln \left(z^{-}-y^{-}\right)
$$

The catch is that the would-be holomorphic and antiholomorphic sectors have a propagator with opposite sign, similar to Siegel's proposal of [5]. The demonstration of (3.18) is straightforward. From the second line of (3.14), it is easy to see that the singular terms 
contributing to the OPE can be organized as

$$
\begin{aligned}
\bar{X}^{m}\left(z^{+}, z^{-}\right) \bar{X}^{n}\left(y^{+}, y^{-}\right) \sim & \frac{1}{4 \mathcal{T}} \sum_{k=1}^{\infty} \frac{1}{k !}(i \bar{y} \mathcal{T})^{k}\left\{\left[X^{m}(z+i \mathcal{T} \bar{z})+X^{m}(z-i \mathcal{T} \bar{z})\right]\left[\partial^{k-1} P^{n}(y)\right]\right\} \\
& -\frac{1}{4 \mathcal{T}} \sum_{k=1}^{\infty} \frac{1}{k !}(-i \bar{y} \mathcal{T})^{k}\left\{\left[X^{m}(z+i \mathcal{T} \bar{z})+X^{m}(z-i \mathcal{T} \bar{z})\right]\left[\partial^{k-1} P^{n}(y)\right]\right\} \\
& +\frac{1}{4 \mathcal{T}} \sum_{k=1}^{\infty} \frac{1}{k !}(i \bar{z} \mathcal{T})^{k}\left\{\left[\partial^{k-1} P^{m}(z)\right]\left[X^{n}(y+i \mathcal{T} \bar{y})+X^{n}(y-i \mathcal{T} \bar{y})\right]\right\} \\
& -\frac{1}{4 \mathcal{T}} \sum_{k=1}^{\infty} \frac{1}{k !}(-i \bar{z} \mathcal{T})^{k}\left\{\left[\partial^{k-1} P^{m}(z)\right]\left[X^{n}(y+i \mathcal{T} \bar{y})+X^{n}(y-i \mathcal{T} \bar{y})\right]\right\}
\end{aligned}
$$

Next, using the OPE

$$
X^{m}(z) P_{n}^{ \pm}(y) \sim \frac{\delta_{n}^{m}}{(z-y)},
$$

the equation above can be rewritten as

$$
\begin{aligned}
\bar{X}^{m}\left(z^{+}, z^{-}\right) \bar{X}^{n}\left(y^{+}, y^{-}\right) \sim & \frac{\eta^{m n}}{4 \mathcal{T}} \sum_{k=1}^{\infty} \frac{1}{k}(i \bar{y} \mathcal{T})^{k}\left\{\frac{1}{(z+i \mathcal{T} \bar{z}-y)^{k}}+\frac{1}{(z-i \mathcal{T} \bar{z}-y)^{k}}\right\} \\
& -\frac{\eta^{m n}}{4 \mathcal{T}} \sum_{k=1}^{\infty} \frac{1}{k}(-i \bar{y} \mathcal{T})^{k}\left\{\frac{1}{(z+i \mathcal{T} \bar{z}-y)^{k}}+\frac{1}{(z-i \mathcal{T} \bar{z}-y)^{k}}\right\} \\
& +\frac{\eta^{m n}}{4 \mathcal{T}} \sum_{k=1}^{\infty} \frac{1}{k}(i \bar{z} \mathcal{T})^{k}\left\{\frac{1}{(y+i \mathcal{T} \bar{y}-z)^{k}}+\frac{1}{(y-i \mathcal{T} \bar{y}-z)^{k}}\right\} \\
& -\frac{\eta^{m n}}{4 \mathcal{T}} \sum_{k=1}^{\infty} \frac{1}{k}(-i \bar{z} \mathcal{T})^{k}\left\{\frac{1}{(y+i \mathcal{T} \bar{y}-z)^{k}}+\frac{1}{(y-i \mathcal{T} \bar{y}-z)^{k}}\right\}
\end{aligned}
$$

These series correspond to the Taylor expansion of logatithm functions. For example,

$$
\sum_{k=1}^{\infty} \frac{1}{k} \frac{(i \bar{y} \mathcal{T})^{k}}{(z+i \mathcal{T} \bar{z}-y)^{k}}=\ln (z+i \mathcal{T} \bar{z}-y+i \mathcal{T} \bar{y})-\ln (z+i \mathcal{T} \bar{z}-y)
$$

Therefore, after some trivial cancellations, (3.18) is obtained. Reproducing such structure in a chiral model ( $\beta \rightarrow \infty$ limit in Siegel's gauge family) was only possible due to the introduction of the sector-splitting operator.

\subsection{Integrated vertex operator and tree level amplitudes}

Having introduced the sector-splitting operator, the next step is to analyze its action on the unintegrated vertex operators of the form (3.1):

$$
\begin{aligned}
\bar{U}\left(z^{+}, z^{-} ; k\right) & \equiv \bar{\Delta} \cdot U(z ; k) \\
& =U_{+}\left(z^{+}\right) U_{-}\left(z^{-}\right) e^{i k \cdot \bar{X}\left(z^{+}, z^{-}\right)} .
\end{aligned}
$$


As a consequence,

$$
U(z ; k)=\lim _{z^{ \pm} \rightarrow z} \bar{U}\left(z^{+}, z^{-} ; k\right) .
$$

Using the equations (3.16), it is then trivial to map any vertex operator in (the chiral limit of) Siegel's string to the sectorized model and vice-versa.

The integrated vertex operator in the sectorized model will be defined as

$$
V(z, \bar{z})=\left(b_{-}\right)_{-1} \cdot\left(b_{+}\right)_{-1} \cdot \bar{\Delta} \cdot U,
$$

and satisfies

$$
\begin{aligned}
{\left[L_{0}, V\right]=} & V+\bar{\partial}(\bar{z} V), \\
{\left[\bar{L}_{0}, V\right]=} & V-\bar{\partial}(\bar{z} V), \\
{[Q, V]=} & \frac{1}{2}\left\{Q,\left(b_{+}+b_{-}\right)_{-1}\right\} \cdot\left(b_{+}-b_{-}\right)_{-1} \cdot \bar{U} \\
& -\frac{1}{2}\left(b_{+}+b_{-}\right)_{-1} \cdot\left\{Q,\left(b_{+}-b_{-}\right)_{-1}\right\} \cdot \bar{U} \\
= & \partial\left\{\frac{1}{2}\left(b_{+}-b_{-}\right)_{-1} \cdot \bar{U}\right\}+i \bar{\partial}\left\{\frac{1}{2}\left(b_{+}+b_{-}\right)_{-1} \cdot \bar{U}\right\},
\end{aligned}
$$

where $\bar{L}_{0}=-\bar{z} \bar{\partial}$ and the $\bar{\Delta}$ properties (3.8) were used. Inside the moduli space integration, $V$ has the expected conformal transformation and its BRST transformation can be written in terms of boundary contributions that vanish upon integration over compact Riemann surfaces.

Naturally, the tensionless limit of this operator should agree with $[1,20]$. In particular, it would be interesting to reproduce Mason and Skinner's $\bar{\delta}(k \cdot P)$ insertions which impose the scattering equations. Through equation (3.14), it follows that the tensionless limit of $\bar{X}_{m}$ is given by $X_{m}(z)+i \bar{z} P_{m}(z)$. Consequently,

$$
\lim _{\mathcal{T} \rightarrow 0} \bar{\Delta} \cdot e^{i k \cdot X}=: e^{i k \cdot X} e^{-\bar{z}(k \cdot P)}: .
$$

When integrated over $\bar{z}$, the second exponential can be thought of as a representation for $\bar{\delta}(k \cdot P)$ and the integrated vertex (3.25) has the expected ambitwistor string limit.

At tree level, $N$-point amplitudes can be cast as

$$
\mathcal{A}_{N}\left(k^{1}, \ldots, k^{N}\right)=\left\langle\prod_{i=1}^{3} U_{i}\left(z_{i} ; k^{i}\right) \prod_{j=4}^{N} \mathcal{V}_{j}\left(k^{j}\right)\right\rangle,
$$

where

$$
\begin{aligned}
\mathcal{V}_{j}\left(k^{j}\right) & \equiv \int_{S^{2}} d^{2} z_{j} V\left(z_{j}, \bar{z}_{j} ; k^{j}\right), \\
& =\frac{1}{2 \mathcal{T}} \int_{S^{2}} d z_{j}^{+} d z_{j}^{-} V^{\prime}\left(z_{j}^{+}, z_{j}^{-} ; k^{j}\right),
\end{aligned}
$$

and $V^{\prime}=V$ when $z_{j}^{ \pm}=z_{j} \pm i \mathcal{T} \bar{z}_{j}$. Because of the point-splitting generated by the operator $\bar{\Delta}$, it might be more convenient to express $\mathcal{V}_{j}$ in terms of the integration over $z_{j}^{ \pm}$, but this is valid only for $\mathcal{T} \neq 0$. 
The actual computation of $\mathcal{A}_{N}$ is very similar to the one in ordinary string theory and, in fact, identical to the amplitudes of Huang, Siegel and Yuan in [12]. The demonstration goes as follows. Consider the unintegrated vertex operators (3.23) and

$$
\overline{\mathcal{A}}_{N}\left(k^{1}, \ldots, k^{N}\right) \equiv\left\langle\prod_{i=1}^{3} \bar{U}_{i}\left(z_{i}^{+}, z_{i}^{-} ; k^{i}\right) \prod_{j=4}^{N} \mathcal{V}_{j}\left(k^{j}\right)\right\rangle .
$$

In this amplitude, the plus and minus sectors behave like the usual holomorphic and antiholomorphic sectors of the string. Using equations (3.16), any vertex in the sectorized description can be mapped to Siegel's string in the chiral limit. From the conformal field theory point of view, evaluating $\overline{\mathcal{A}}_{N}\left(k^{1}, \ldots, k^{n}\right)$ using the $\left(P_{m}, X^{m}\right)$ first order action is equivalent to the same computation using the second order action for $\bar{X}^{m}$ with propagator (3.18). Since $\overline{\mathcal{A}}_{N}$ is Möbius invariant, i.e. independent of the fixed coordinates $z_{i}^{ \pm}$for $i=1,2,3$, setting $z_{i}^{+}=z_{i}^{-}$would then recover the amplitude $\mathcal{A}_{N}$. The only difference is that in the chiral model there are no $\beta$-modified propagators.

It might be useful to analyze the $\left(P_{m}, X^{m}\right)$ contribution to $\mathcal{A}_{N}\left(k^{1}, \ldots, k^{N}\right)$. Through the equations (3.16) and the OPE (3.18), it is easy to show that

$$
\bar{X}_{m}\left(z^{+}, z^{-}\right) P_{n}^{ \pm}(y) \sim \frac{\eta_{m n}}{\left(z^{ \pm}-y\right)}
$$

which can be used to compute the OPE reduction of $\mathcal{A}_{N}\left(k^{1}, \ldots, k^{N}\right)$ by contracting all the operators $P_{m}^{ \pm}$with themselves and with the exponentials. Finally, the remaining contribution involves only plane-wave operators and can be generically written as

$$
\mathcal{A}_{N}^{X}(z, \bar{z}, k)=\left\langle\prod_{i=1}^{3}: e^{i k^{i} \cdot X\left(z_{i}\right)}: \prod_{j=4}^{N}: e^{i k^{j} \cdot \bar{X}\left(z_{j}, \bar{z}_{j}\right)}:\right\rangle .
$$

There are two simple ways for evaluating this contribution. The first is to analyze instead

$$
\overline{\mathcal{A}}_{N}^{X}\left(z^{+}, z^{-}, k\right)=\left\langle\prod_{i=1}^{N}: e^{i k^{i} \cdot \bar{X}\left(z_{i}^{+}, z_{i}^{-}\right)}:\right\rangle,
$$

using the propagator (3.18). The zero-mode integration of $X^{m}$ gives the usual momentum conservation delta,

$$
\delta^{d}\left(\sum_{i=1}^{N} k_{i}^{m}\right)
$$

and the final result can be sketched as

$$
\overline{\mathcal{A}}_{N}^{X}\left(z^{+}, z^{-}, k\right) \propto \delta^{d}\left(\sum k\right) \prod_{i>j}^{N}\left(\frac{z_{i j}^{+}}{z_{i j}^{-}}\right)^{\frac{\left(k_{i} \cdot k_{j}\right)}{2 \mathcal{T}}} .
$$

As explained above, $\mathcal{A}_{N}^{X}$ is recovered for $z_{i}^{+}=z_{i}^{-}$and $i=1,2,3$. 
Alternatively, $\mathcal{A}_{N}^{X}$ can be computed in a similar way to the standard ambitwistor construction. By incorporating the exponentials in the path integral action, it can be cast as

$$
\begin{gathered}
S[X, P]=\int d^{2} z\left\{\frac{1}{2 \pi} P_{m} \bar{\partial} X^{m}+i \sum_{i=1}^{3} k_{m}^{i} X^{m} \delta^{2}\left(z-z_{i}\right)+\frac{i}{2} \sum_{j=4}^{N} k_{m}^{j} X^{m}\left[\delta^{2}\left(z-z_{j}^{+}\right)+\delta^{2}\left(z-z_{j}^{-}\right)\right]\right. \\
\left.-\bar{z} \sum_{j=4}^{N} \sum_{n=0}^{\infty} \frac{(i \bar{z} \mathcal{T})^{2 n}}{(2 n+1) !} k^{j m} \partial^{2 n} P_{m}(z) \delta^{2}\left(z-z_{j}\right)\right\},
\end{gathered}
$$

where equation (3.14) was used. Again, the zero-mode integration of $X^{m}$ gives (3.34), while the integration of the non-zero modes imply that

$$
\frac{1}{2 \pi} \bar{\partial} P_{m}=i \sum_{i=1}^{3} k_{m}^{i} \delta^{2}\left(z-z_{i}\right)+\frac{i}{2} \sum_{j=4}^{N} k_{m}^{j}\left[\delta^{2}\left(z-z_{j}-i \mathcal{T} \bar{z}_{j}\right)+\delta^{2}\left(z-z_{j}+i \mathcal{T} \bar{z}_{j}\right)\right] .
$$

On the Riemann sphere, there is a unique solution for this equation, given by

$$
P^{m}(z)=i \sum_{i=1}^{3} \frac{k_{i}^{m}}{\left(z-z_{i}\right)}+i \sum_{j=4}^{N}\left(\frac{\frac{1}{2} k_{j}^{m}}{\left(z-z_{j}-i \mathcal{T} \bar{z}_{j}\right)}+\frac{\frac{1}{2} k_{j}^{m}}{\left(z-z_{j}+i \mathcal{T} \bar{z}_{j}\right)}\right),
$$

which has the expected tensionless limit. Because of the point-splitting, $P_{m}\left(z_{j}\right)$ is not singular for $\mathcal{T} \neq 0$. It is then straightforward to show that the amplitude contribution of the action $S[X, P]$ is equal to (3.35) when $z_{i}^{+}=z_{i}^{-}$. In particular, the Koba-Nielsen like factors of the amplitude are generated by replacing the solution (3.38) on the second line of (3.36).

\section{Final remarks}

The results presented here establish a simple realization of the fact that tensile chiral strings can be effectively seen as ordinary strings in the conformal gauge with a relative sign flip between the left and right moving parts of the $\langle X X\rangle$ propagator. This idea was proposed by Siegel in [5] and interpreted as a change in the boundary conditions of the model.

The sectorized description of [18], therefore, was an incomplete manifestation of this equivalence. The missing link, presented here in section 3, was the explicit construction of the integrated vertex operators in the chiral model.

At genus $0, N$-point amplitudes computed using either the new definition of the integrated vertex operator or the Siegel's sign-flipped model are equivalent. At the loop level, on the other hand, there might be some subtleties. The modular invariance of the type II ambitwistor string at one-loop was shown in [14]. However, for the bosonic case in the null string framework, one-loop modular invariance was not observed [16]. Using the sign-flipped model, this result was confirmed for tensile chiral strings [17]. It might be interesting to investigate these results within the framework presented here. In particular, a better understanding of the geometrical meaning of Siegel's gauge family for the torus and higher genus Riemann surfaces should be useful. It is not obvious, for example, that the mentioned equivalence between the tensile chiral string and the ordinary string with the sign flipped propagator holds at loop level. The reason is that the point-splitting generated 
by the operator $\bar{\Delta}$ is invisible to the modular parameter. In other words, the would-be left and right moving propagators depend on the same modular parameter, $\tau$, as opposite to the usual $\tau$ and $\bar{\tau}$. An appropriate loop prescription should be found and, in this direction, the results of [23] may be helpful.

The solutions (3.38) give rise to modified scattering equations that should also accommodate massive particles. The momenta $k_{j}(j \geq 4)$ have a split structure, each one virtually behaving as two particles with half of the total momentum located on the Riemann sphere at $z_{j} \pm i \mathcal{T} \bar{z}_{j}$. However, this idea has not yet been investigated and will be left to a future work. The possible outcomes shall be compared with known results in the literature of scattering equations of massive particles, e.g. [26].

\section{Acknowledgments}

I would like to thank Matheus Lize, and especially Thales Azevedo and the anonymous referee from JHEP for useful comments and suggestions. I would like to thank also the Galileo Galilei Institute for Theoretical Physics and INFN for their hospitality and support during the workshop "String Theory from a worldsheet perspective", where part of this work has been done. This research has been supported by the Czech Science Foundation - GAČR, project 19-06342Y.

\section{A A concrete example: the bosonic chiral string}

In this appendix, the ideas developed in the main text will be applied to the bosonic chiral string, with BRST charge (2.10). The cohomology will be reviewed with a subsequent evaluation of all tree-level 3-point amplitudes and the 4-point amplitudes with massless external states. The latter is in agreement with the results of [27].

\section{A.1 Cohomology}

The BRST cohomology at ghost number zero is given by the identity operator. At ghost number one, the cohomology contains only the zero-momentum operators $c_{+} P_{m}^{+}$and $c_{-} P_{m}^{-}$.

Physical states are defined as elements of the BRST cohomology with ghost number two, conformal weight zero and annihilated by the zero mode of $b=b_{+}+b_{-}$. They can be generically expressed as $U=U_{0}+U_{+}+U_{-}$, such that

$$
\begin{aligned}
U_{0}= & c_{+} c_{-} P_{m}^{+} P_{n}^{-} A^{m n}+\mathcal{T}\left(c_{+} \partial^{2} c_{+}+c_{-} \partial^{2} c_{-}\right) A+\mathcal{T}\left(c_{+} \partial^{2} c_{+}-c_{-} \partial^{2} c_{-}\right) B \\
& +c_{+} P_{m}^{+}\left(\partial c_{+}-\partial c_{-}\right) A^{m}+c_{-} P_{m}^{-}\left(\partial c_{+}-\partial c_{-}\right) B^{m}, \\
U_{+}= & c_{+} c_{-} P_{m}^{+} P_{n}^{+} C_{+}^{m n}+c_{-} P_{m}^{+}\left(\partial c_{+}-\partial c_{-}\right) C_{+}^{m}+c_{+} c_{-} \partial P_{m}^{+} D_{+}^{m} \\
& +\mathcal{T} c_{-} \partial^{2} c_{+} C^{+}+b_{+} c_{+} c_{-}\left(\partial c_{+}-\partial c_{-}\right) D^{+} \\
U_{-}= & c_{+} c_{-} P_{m}^{-} P_{n}^{-} C_{-}^{m n}+c_{+} P_{m}^{-}\left(\partial c_{+}-\partial c_{-}\right) C_{-}^{m}-c_{+} c_{-} \partial P_{m}^{-} D_{-}^{m} \\
& +\mathcal{T} c_{+} \partial^{2} c_{-} C^{-}+b_{-} c_{+} c_{-}\left(\partial c_{+}-\partial c_{-}\right) D^{-} .
\end{aligned}
$$


$U_{0}$ describes massless fields $\left(A^{m n}, A^{m}, B^{m}, A\right.$ and $B$ ), while $U_{ \pm}$describes fields of mass $m^{2}= \pm 4 \mathcal{T}\left(C_{ \pm}^{m n}, C_{ \pm}^{m}, D_{ \pm}^{m}, C^{ \pm}\right.$and $\left.D^{ \pm}\right)$. The equations of motion are given by

$$
\begin{aligned}
& \square A^{m n}=2\left(\partial^{n} A^{m}+\partial^{m} B^{n}\right), \quad(\square \mp 4 \mathcal{T}) C_{ \pm}^{m n}=\partial^{m} C_{ \pm}^{n}+\partial^{n} C_{ \pm}^{m} \mp \eta^{m n} D^{ \pm}, \\
& \square A=\partial_{m}\left(A^{m}+B^{m}\right) \quad(\square \mp 4 \mathcal{T}) C^{ \pm}=2 \partial_{m} C_{ \pm}^{m} \mp 6 D^{ \pm}, \\
& \square B=\partial_{m}\left(A^{m}-B^{m}\right), \quad(\square \mp 4 \mathcal{T}) D_{ \pm}^{m}=4 \mathcal{T} C_{ \pm}^{m}+2 \partial^{m} D^{ \pm}, \\
& A^{m}=\frac{1}{2}\left[\partial_{n} A^{m n}-\partial^{m}(A-B)\right], \quad C_{ \pm}^{m} \pm D_{ \pm}^{m}=\partial_{n} C_{ \pm}^{m n}-\frac{1}{2} \partial^{m} C^{ \pm}, \\
& B^{m}=\frac{1}{2}\left[\partial_{n} A^{n m}-\partial^{m}(A+B)\right], \quad D^{ \pm}-\frac{1}{2} \partial_{m} D_{ \pm}^{m}=\frac{\mathcal{T}}{2}\left(C_{ \pm}^{m n} \eta_{m n}-3 C^{ \pm}\right),
\end{aligned}
$$

with gauge transformations

$$
\begin{aligned}
& \delta A^{m n}=\partial^{n} \alpha^{m}+\partial^{m} \beta^{n}, \quad \delta C_{ \pm}^{m n}=\partial^{m} \lambda_{ \pm}^{n}+\partial^{n} \lambda_{ \pm}^{m} \mp \eta^{m n} \sigma^{ \pm}, \\
& \delta A^{m}=\frac{1}{2} \square \alpha^{m}+\frac{1}{2} \partial^{m} \omega, \quad \delta C_{ \pm}^{m}=(\square \mp 4 \mathcal{T}) \lambda_{ \pm}^{m}, \\
& \delta B^{m}=\frac{1}{2} \square \beta^{m}-\frac{1}{2} \partial^{m} \omega, \quad \delta D_{ \pm}^{m}=2 \partial^{m} \sigma^{ \pm}+4 \mathcal{T} \lambda_{ \pm}^{m}, \\
& \delta A=\frac{1}{2} \partial_{m}\left(\alpha^{m}+\beta^{m}\right), \quad \delta C^{ \pm}=2 \partial_{m} \lambda_{ \pm}^{m} \mp 6 \sigma^{ \pm}, \\
& \delta B=\omega+\frac{1}{2} \partial_{m}\left(\alpha^{m}-\beta^{m}\right), \quad \delta D^{ \pm}=(\square \mp 4 \mathcal{T}) \sigma^{ \pm} .
\end{aligned}
$$

Here, $\alpha^{m}, \beta^{m}, \omega, \lambda_{ \pm}^{m}$ and $\sigma^{ \pm}$are the gauge parameters.

In order to make the physical degrees of freedom more transparent, it is convenient to make some field redefinitions. For the massless vertex $U_{0}$, consider

$$
\begin{array}{rlrl}
g^{m} & \equiv A^{m}+B^{m}-\partial^{m} A, & b^{m} & \equiv A^{m}-B^{m}-\partial^{m} B, \\
g^{m n} & \equiv \frac{1}{2}\left(A^{m n}+A^{n m}\right), & b^{m n} & \equiv \frac{1}{2}\left(A^{m n}-A^{n m}\right), \\
\phi & \equiv \mathcal{T}\left(\frac{1}{2} A^{m n} \eta_{m n}-A\right), \quad b^{m n p} & \equiv \frac{1}{3}\left(\partial^{p} b^{m n}+\partial^{m} b^{n p}+\partial^{n} b^{p m}\right),
\end{array}
$$

such that the equations of motion above are rewritten as

$$
\begin{aligned}
g^{m} & =\partial_{n} g^{m n}-\eta_{n p} \partial^{m} g^{n p}+\frac{2}{\mathcal{T}} \partial^{m} \phi, & b^{m} & =\partial_{n} b^{m n}, \\
\square g^{m n} & =\partial_{p} \partial^{n} g^{m p}+\partial_{p} \partial^{m} g^{n p}-\eta_{p q} \partial^{m} \partial^{n} g^{p q}+\frac{2}{\mathcal{T}} \partial^{m} \partial^{n} \phi, & \partial_{p} b^{m n p} & =0, \\
\square \phi & =0, & &
\end{aligned}
$$

with gauge transformations

$$
\begin{aligned}
\delta \phi & =0, \\
\delta g^{m n} & =\frac{1}{4} \partial^{n}\left(\alpha^{m}+\beta^{m}\right)+\frac{1}{4} \partial^{m}\left(\alpha^{n}+\beta^{n}\right), \\
\delta b^{m n} & =\frac{1}{4} \partial^{n}\left(\alpha^{m}-\beta^{m}\right)-\frac{1}{4} \partial^{m}\left(\alpha^{n}-\beta^{n}\right) .
\end{aligned}
$$

$\phi$ corresponds to the dilaton, $b^{m n}$ is the Kalb-Ramond 2 -form and $g^{m n}$ is the graviton. 
For the vertices $U_{+}$and $U_{-}$, consider the combinations

$$
\begin{aligned}
d_{ \pm}^{m n} \equiv & C_{ \pm}^{m n}-\frac{1}{4 \mathcal{T}}\left(\partial^{n} D_{ \pm}^{m}+\partial^{m} D_{ \pm}^{n}\right) & d_{ \pm}^{m} \equiv D_{ \pm}^{m} \pm \frac{1}{10}\left(\eta_{n p} \partial^{m} C_{ \pm}^{n p}-\partial^{m} C^{ \pm}\right) \\
& \pm \frac{1}{20 \mathcal{T}}\left(\partial^{m} \partial^{n} \pm \mathcal{T} \eta^{m n}\right)\left(C^{ \pm}-C_{ \pm}^{p q} \eta_{p q}\right) & d_{ \pm} \equiv C^{ \pm}-C_{ \pm}^{m n} \eta_{m n}
\end{aligned}
$$

with gauge transformations

$$
\delta d_{ \pm}= \pm 20 \sigma^{ \pm}, \quad \delta d_{ \pm}^{m}=4 \mathcal{T} \lambda_{ \pm}^{m}, \quad \delta d_{ \pm}^{m n}=0 .
$$

The fields $d_{ \pm}$and $d_{ \pm}^{m}$ are pure gauge, while $d_{ \pm}^{m n}$ corresponds to spin 2 fields with $m^{2}= \pm 4 \mathcal{T}$ satisfying

$$
(\square \mp 4 \mathcal{T}) d_{ \pm}^{m n}=0, \quad \partial_{n} d_{ \pm}^{m n}=0, \quad d_{ \pm}^{m n} \eta_{m n}=0 .
$$

\section{A.2 3-point amplitudes at tree level}

For the amplitude evaluations, it is simpler to consider a gauge fixed form of the vertices above with momentum eigenstates. In addition, it might be helpful to work with their sector-split versions, cf. definition (3.23), in order to build some intuition on the 3-point amplitudes and their independence from the fixed positions of the vertices (Möbius invariance). The gauge fixed vertices will be chosen to be:

- massless vertex, $U_{0}$, with sector-split form

$$
\bar{U}_{0}\left(z^{+}, z^{-} ; k_{m}\right)=A^{m n} c_{+} P_{m}^{+}\left(z^{+}\right) c_{-} P_{n}^{-}\left(z^{-}\right) e^{i k \cdot \bar{X}\left(z^{+}, z^{-}\right)},
$$

with $k_{m} k^{m}=0, k_{n} A^{m n}=k_{n} A^{n m}=0$;

- massive vertex, $U_{+}$, with sector-split form

$$
\bar{U}_{+}\left(z^{+}, z^{-} ; k_{m}\right)=C_{+}^{m n} c_{+} P_{m}^{+} P_{n}^{+}\left(z^{+}\right) c_{-}\left(z^{-}\right) e^{i k \cdot \bar{X}\left(z^{+}, z^{-}\right)}
$$

with $k_{m} k^{m}=-4 \mathcal{T}, k_{n} C_{+}^{m n}=0$ and $C_{+}^{m n} \eta_{m n}=0$;

- and the tachyonic vertex, $U_{-}$, with sector-split form

$$
\bar{U}_{-}\left(z^{+}, z^{-} ; k_{m}\right)=C_{-}^{m n} c_{+}\left(z^{+}\right) c_{-} P_{m}^{-} P_{n}^{-}\left(z^{-}\right) e^{i k \cdot \bar{X}\left(z^{+}, z^{-}\right)},
$$

with $k_{m} k^{m}=4 \mathcal{T}, k_{n} C_{-}^{m n}=0$ and $C_{-}^{m n} \eta_{m n}=0$;

There are ten 3-point tree level amplitudes that can be built from $U_{0}$ and $U_{ \pm}$, which will be generically cast as

$$
\mathcal{A}_{3}=\left\langle\prod_{i=1}^{3} \bar{U}_{i}\left(z_{i}^{+}, z_{i}^{-} ; k_{m}^{i}\right)\right\rangle .
$$

The contributions from the ghost measures are simply

$$
\left\langle c_{ \pm}\left(z_{1}^{ \pm}\right) c_{ \pm}\left(z_{2}^{ \pm}\right) c_{ \pm}\left(z_{3}^{ \pm}\right)\right\rangle=z_{12}^{ \pm} z_{23}^{ \pm} z_{31}^{ \pm},
$$


where $z_{i j}^{ \pm}=z_{i}^{ \pm}-z_{j}^{ \pm}$. The matter contribution is obtained with the OPE reduction of the amplitude by computing all the contractions of the $P_{m}^{ \pm}$'s with themselves and with the exponentials $e^{i k \cdot \bar{X}}$. At the end, the remaining contribution from the exponentials is given by equation (3.35). For 3-point amplitudes, it will be defined as

$$
\begin{aligned}
I_{3}\left(k, z^{+}, z^{-}\right) & \equiv\left\langle: e^{i k^{1} \cdot \bar{X}\left(z_{1}^{+}, z_{1}^{-}\right)}:: e^{i k^{2} \cdot \bar{X}\left(z_{2}^{+}, z_{2}^{-}\right)}:: e^{i k^{3} \cdot \bar{X}\left(z_{3}^{+}, z_{3}^{-}\right)}:\right\rangle \\
& =\delta^{26}\left(k^{1}+k^{2}+k^{3}\right)\left(\frac{z_{12}^{+}}{z_{12}^{-}}\right)^{\frac{\left(k_{1} \cdot k_{2}\right)}{2 \mathcal{T}}}\left(\frac{z_{23}^{+}}{z_{23}^{-}}\right)^{\frac{\left(k_{2} \cdot k_{3}\right)}{2 \mathcal{T}}}\left(\frac{z_{31}^{+}}{z_{31}^{-}}\right)^{\frac{\left(k_{3} \cdot k_{1}\right)}{2 \mathcal{T}}} .
\end{aligned}
$$

Although $z_{i j}^{+}$and $z_{i j}^{-}$appear with opposite powers, this is precisely the combination needed to show the Möbius invariance of the amplitudes. The list of all 3-point amplitudes can be cast as:

$$
\begin{aligned}
& \mathcal{A}_{000}=A^{m \bar{m}} A^{n \bar{n}} A^{p \bar{p}} T_{m n p}^{+} T_{\bar{m} \bar{n} \bar{p}}^{-} \delta^{26}\left(k^{1}+k^{2}+k^{3}\right), \\
& \mathcal{A}_{00+}=\left(k_{\bar{m}}^{3} k_{\bar{n}}^{3}+2 \mathcal{T} \eta_{\bar{m} \bar{n}}\right) D_{m n p q}^{+} A^{m \bar{m}} A^{n \bar{n}} C_{+}^{p q} \delta^{26}\left(k^{1}+k^{2}+k^{3}\right) \text {, } \\
& \mathcal{A}_{00-}=\left(k_{m}^{3} k_{n}^{3}-2 \mathcal{T} \eta_{m n}\right) D_{\bar{m} \bar{n} \bar{p} \bar{q}}^{-} A^{m \bar{m}} A^{n \bar{n}} C_{-}^{\bar{p} \bar{q}} \delta^{26}\left(k^{1}+k^{2}+k^{3}\right) \text {, } \\
& \mathcal{A}_{0++}=\frac{1}{2}\left(k_{\bar{m}}^{2}-k_{\bar{m}}^{3}\right) E_{m n p q r}^{+} A^{m \bar{m}} C_{+}^{n p} C_{+}^{q r} \delta^{26}\left(k^{1}+k^{2}+k^{3}\right) \text {, } \\
& \mathcal{A}_{0--}=\frac{1}{2}\left(k_{m}^{2}-k_{m}^{3}\right) E_{\bar{m} \bar{n} \bar{p} \bar{q}}^{-} A^{m \bar{m}} C_{-}^{\bar{n} \bar{p}} C_{-}^{\bar{q} \bar{r}} \delta^{26}\left(k^{1}+k^{2}+k^{3}\right), \\
& \mathcal{A}_{0+-}=F_{m n p}^{+} F_{\bar{m} \bar{n} \bar{p}}^{-} A^{m \bar{m}} C_{+}^{n p} C_{-}^{\bar{n} \bar{p}} \delta^{26}\left(k^{1}+k^{2}+k^{3}\right), \\
& \mathcal{A}_{++-}=k_{\bar{m}}^{1} k_{\bar{n}}^{2} G_{m n p q}^{+} C_{+}^{m n} C_{+}^{p q} C_{-}^{\bar{m} \bar{n}} \delta^{26}\left(k^{1}+k^{2}+k^{3}\right) \text {, } \\
& \mathcal{A}_{+--}=k_{m}^{1} k_{n}^{2} G_{\bar{m} \bar{n} \bar{p} \bar{q}}^{-} C_{-}^{\bar{m} \bar{n}} C_{-}^{\bar{p} \bar{q}} C_{+}^{m n} \delta^{26}\left(k^{1}+k^{2}+k^{3}\right), \\
& \mathcal{A}_{+++}=H_{\text {mnpqrs }}^{+} C_{+}^{m n} C_{+}^{p q} C_{+}^{r s} \delta^{26}\left(k^{1}+k^{2}+k^{3}\right) \text {, } \\
& \mathcal{A}_{---}=H_{\bar{m} \bar{n} \bar{p} \bar{q} \bar{r} \bar{s}}^{-} C_{-}^{\bar{m} \bar{n}} C_{-}^{\bar{p} \bar{q}} C_{-}^{\bar{r} \bar{s}} \delta^{26}\left(k^{1}+k^{2}+k^{3}\right),
\end{aligned}
$$

where $A_{m \bar{m}}, C_{m n}^{+}$and $C_{\bar{m} \bar{n}}^{-}$are the vertex polarizations as presented above and the kinematic tensors are given by

$$
\begin{aligned}
T_{m n p}^{ \pm} \equiv & k_{m}^{2} k_{n}^{3} k_{p}^{1} \pm 2 \mathcal{T}\left(k_{m}^{2} \eta_{n p}+k_{n}^{3} \eta_{m p}+k_{p}^{1} \eta_{m n}\right), \\
D_{m n p q}^{ \pm} \equiv & 8 \mathcal{T}^{2} \eta_{m p} \eta_{n q} \mp 2 \mathcal{T}\left(2 \eta_{m p} k_{n}^{1} k_{q}^{1}+2 \eta_{n p} k_{m}^{2} k_{q}^{2}-\eta_{m n} k_{p}^{1} k_{q}^{2}\right)-k_{p}^{1} k_{q}^{2} k_{m}^{3} k_{n}^{3}, \\
E_{m n p q r}^{ \pm} \equiv & 4 \mathcal{T} \eta_{m n}\left(4 \mathcal{T} \eta_{p q} \mp k_{p}^{1} k_{q}^{1}\right) k_{r}^{1}-4 \mathcal{T} \eta_{m q}\left(4 \mathcal{T} \eta_{n r} \mp k_{n}^{1} k_{r}^{1}\right) k_{p}^{1} \\
& +\frac{1}{4}\left(k_{m}^{2}-k_{m}^{3}\right)\left(4 \mathcal{T} \eta_{n r} \mp k_{n}^{1} k_{r}^{1}\right)\left(4 \mathcal{T} \eta_{p q} \mp k_{p}^{1} k_{q}^{1}\right) \\
& \mp \mathcal{T}\left(k_{m}^{2}-k_{m}^{3}\right)\left(\eta_{p q} k_{n}^{1} k_{r}^{1}+\eta_{n r} k_{p}^{1} k_{q}^{1}\right)+\frac{1}{4}\left(k_{m}^{2}-k_{m}^{3}\right) k_{n}^{1} k_{r}^{1} k_{p}^{1} k_{q}^{1}, \\
F_{m n p}^{ \pm} \equiv & k_{n}^{1} k_{p}^{1} k_{m}^{3} \pm 2 \mathcal{T}\left(\eta_{m n} k_{p}^{1}+\eta_{m p} k_{n}^{1}\right), \\
G_{m n p q}^{ \pm} \equiv & 4 \mathcal{T}^{2}\left(\eta_{m p} \eta_{n q}+\eta_{m q} \eta_{n p}\right)-k_{m}^{3} k_{n}^{3} k_{p}^{3} k_{q}^{3} \\
& \mp 2 \mathcal{T}\left(\eta_{m p} k_{n}^{3} k_{q}^{3}+\eta_{m q} k_{n}^{3} k_{p}^{3}+\eta_{n p} k_{m}^{3} k_{q}^{3}+\eta_{n q} k_{m}^{3} k_{p}^{3}\right),
\end{aligned}
$$




$$
\begin{aligned}
H_{m n p q r s}^{ \pm} \equiv & k_{r}^{1} k_{s}^{1} k_{m}^{2} k_{n}^{2} k_{p}^{3} k_{q}^{3} \pm 32 \mathcal{T}^{3}\left(\eta_{m p} \eta_{n r} \eta_{q s}+\eta_{m r} \eta_{n p} \eta_{q s}\right) \\
& +8 \mathcal{T}^{2}\left(2 \eta_{m p} \eta_{n r} k_{q}^{3}+2 \eta_{m r} \eta_{n p} k_{q}^{3}+\eta_{m p} \eta_{n q} k_{r}^{1}\right) k_{s}^{1} \\
& +8 \mathcal{T}^{2}\left(2 \eta_{n p} \eta_{q r} k_{s}^{1}+2 \eta_{n q} \eta_{p r} k_{s}^{1}+\eta_{p r} \eta_{q s} k_{n}^{2}\right) k_{m}^{2} \\
& +8 \mathcal{T}^{2}\left(2 \eta_{n r} \eta_{p s} k_{m}^{2}+2 \eta_{n s} \eta_{p r} k_{m}^{2}+\eta_{m r} \eta_{n s} k_{p}^{3}\right) k_{q}^{3} \\
& \pm 2 \mathcal{T}\left(\eta_{p r} k_{s}^{1} k_{q}^{3}+\eta_{p s} k_{r}^{1} k_{q}^{3}+\eta_{q r} k_{s}^{1} k_{p}^{3}+\eta_{q s} k_{r}^{1} k_{p}^{3}\right) k_{m}^{2} k_{n}^{2} \\
& \pm 2 \mathcal{T}\left(\eta_{m r} k_{s}^{1} k_{n}^{2}+\eta_{m s} k_{r}^{1} k_{n}^{2}+\eta_{n r} k_{s}^{1} k_{m}^{2}+\eta_{n s} k_{r}^{1} k_{m}^{2}\right) k_{p}^{3} k_{q}^{3} \\
& \pm 2 \mathcal{T}\left(\eta_{m p} k_{n}^{2} k_{q}^{3}+\eta_{n p} k_{m}^{2} k_{q}^{3}+\eta_{m q} k_{n}^{2} k_{p}^{3}+\eta_{n q} k_{m}^{2} k_{p}^{3}\right) k_{r}^{1} k_{s}^{1}
\end{aligned}
$$

Note, in particular, that the 3 -point amplitude with massless vertices, $\mathcal{A}_{000}$, is in agreement with the results of [12].

\section{A.3 4-point amplitude with massless external states}

As an example, the 4-point amplitude with external massless states will be now analyzed. In addition to Möbius invariance, it will be shown that the amplitude has the expected poles located over the mass spectrum of the physical states.

Consider

$$
\mathcal{A}_{0000}=\left\langle\bar{U}_{0}\left(z_{1}^{+}, z_{1}^{-} ; k^{1}\right) \bar{U}_{0}\left(z_{2}^{+}, z_{2}^{-} ; k^{2}\right) \bar{U}_{0}\left(z_{3}^{+}, z_{3}^{-} ; k^{3}\right) \mathcal{V}_{0}\left(k^{4}\right)\right\rangle
$$

where the integrated vertex operator, $\mathcal{V}_{0}\left(k^{4}\right)$, is given by

$$
\mathcal{V}_{0}\left(k^{4}\right)=\frac{1}{2 \mathcal{T}} A^{m n} \int_{S^{2}} d z^{+} d z^{-} P_{m}^{+}\left(z^{+}\right) P_{n}^{-}\left(z^{-}\right) e^{i k^{4} \cdot \bar{X}\left(z^{+}, z^{-}\right)},
$$

cf. equation (3.29) and the gauge fixed unintegrated vertex operator (A.12).

In this case, the Mandelstam variables are such that

$$
\begin{aligned}
s & \equiv-\left(k^{1}+k^{2}\right)^{2}, \quad t & \equiv-\left(k^{1}+k^{3}\right)^{2}, \quad u & \equiv-\left(k^{1}+k^{4}\right)^{2}, \\
& =-2\left(k^{1} \cdot k^{2}\right), \quad & =-2\left(k^{1} \cdot k^{3}\right), & =-2\left(k^{1} \cdot k^{4}\right),
\end{aligned}
$$

and $s+t+u=0$. Using the results of subsection 3.2, the computation of $\mathcal{A}_{0000}$ is straightforward and can be cast as

$$
\mathcal{A}_{0000}=\frac{1}{2 \mathcal{T}} A^{m \bar{m}} A^{n \bar{n}} A^{p \bar{p}} A^{q \bar{q}} \int_{S^{2}} d z_{4}^{+} d z_{4}^{-}\left\{T_{m n p q}^{+} T_{\bar{m} \bar{n} \bar{p} \bar{q}}^{-} I_{4}\left(k, z^{+}, z^{-}\right)\right\},
$$

where

$$
\begin{aligned}
I_{4}\left(k, z^{+}, z^{-}\right) & \equiv\left\langle: e^{i k^{1} \cdot \bar{X}\left(z_{1}^{+}, z_{1}^{-}\right)}:: e^{i k^{2} \cdot \bar{X}\left(z_{2}^{+}, z_{2}^{-}\right)}:: e^{i k^{3} \cdot \bar{X}\left(z_{3}^{+}, z_{3}^{-}\right)}:: e^{i k^{4} \cdot \bar{X}\left(z_{4}^{+}, z_{4}^{-}\right)}:\right\rangle \\
& =\delta^{26}\left(k^{1}+k^{2}+k^{3}+k^{4}\right)\left(\frac{z_{12}^{+} z_{34}^{+}}{z_{23}^{+} z_{14}^{+}} \frac{z_{23}^{-} z_{14}^{-}}{z_{12}^{-} z_{34}^{-}}\right)^{-\frac{s}{4 \mathcal{T}}}\left(\frac{z_{31}^{+} z_{24}^{+}}{z_{23}^{+} z_{14}^{+}} \frac{z_{23}^{-} z_{14}^{-}}{z_{31}^{-} z_{24}^{-}}\right)^{-\frac{t}{4 \mathcal{T}}}
\end{aligned}
$$


The dynamical tensors $T_{m n p q}^{+}$and $T_{\bar{m} \bar{n} \bar{p} \bar{q}}^{-}$are defined as

$$
\begin{aligned}
T_{m n p q}^{ \pm} \equiv & 4 \mathcal{T}^{2} \eta_{m n} \eta_{p q} \frac{z_{12}^{ \pm} z_{23}^{ \pm} z_{31}^{ \pm}}{\left(z_{12}^{ \pm}\right)^{2}\left(z_{34}^{ \pm}\right)^{2}}+4 \mathcal{T}^{2} \eta_{m p} \eta_{n q} \frac{z_{12}^{ \pm} z_{23}^{ \pm} z_{31}^{ \pm}}{\left(z_{13}^{ \pm}\right)^{2}\left(z_{24}^{ \pm}\right)^{2}}+4 \mathcal{T}^{2} \eta_{m q} \eta_{n p} \frac{z_{12}^{ \pm} z_{23}^{ \pm} z_{31}^{ \pm}}{\left(z_{14}^{ \pm}\right)^{2}\left(z_{23}^{ \pm}\right)^{2}} \\
& \pm 2 \mathcal{T} \eta_{m q} \frac{z_{12}^{ \pm} z_{23}^{ \pm} z_{31}^{ \pm}}{\left(z_{14}^{ \pm}\right)^{2}} V_{n}^{2} V_{p}^{3} \pm 2 \mathcal{T} \eta_{n q} \frac{z_{12}^{ \pm} z_{23}^{ \pm} z_{31}^{ \pm}}{\left(z_{24}^{ \pm}\right)^{2}} V_{m}^{1} V_{p}^{3} \pm 2 \mathcal{T} \eta_{p q} \frac{z_{12}^{ \pm} z_{23}^{ \pm} z_{31}^{ \pm}}{\left(z_{34}^{ \pm}\right)^{2}} V_{m}^{1} V_{n}^{2} \\
& \pm 2 \mathcal{T} \eta_{m n} \frac{z_{12}^{ \pm} z_{23}^{ \pm} z_{31}^{ \pm}}{\left(z_{12}^{ \pm}\right)^{2}} V_{p}^{3} V_{q}^{4} \pm 2 \mathcal{T} \eta_{m p} \frac{z_{12}^{ \pm} z_{23}^{ \pm} z_{31}^{ \pm}}{\left(z_{13}^{ \pm}\right)^{2}} V_{n}^{2} V_{q}^{4} \pm 2 \mathcal{T} \eta_{n p} \frac{z_{12}^{ \pm} z_{23}^{ \pm} z_{31}^{ \pm}}{\left(z_{23}^{ \pm}\right)^{2}} V_{m}^{1} V_{q}^{4} \\
& +z_{12}^{ \pm} z_{23}^{ \pm} z_{31}^{ \pm} V_{m}^{1} V_{n}^{2} V_{p}^{3} V_{q}^{4}
\end{aligned}
$$

with

$$
\begin{array}{rlrl}
V_{m}^{1} \equiv k_{m}^{2} \frac{z_{24}^{ \pm}}{z_{12}^{ \pm} z_{14}^{ \pm}}+k_{m}^{3} \frac{z_{34}^{ \pm}}{z_{13}^{ \pm} z_{14}^{ \pm}}, & V_{q}^{4} \equiv & \frac{1}{3} k_{q}^{1}\left(\frac{z_{12}^{ \pm}}{z_{14}^{ \pm} z_{24}^{ \pm}}-\frac{z_{31}^{ \pm}}{z_{14}^{ \pm} z_{34}^{ \pm}}\right) \\
V_{n}^{2} \equiv k_{n}^{1} \frac{z_{14}^{ \pm}}{z_{21}^{ \pm} z_{24}^{ \pm}}+k_{n}^{3} \frac{z_{34}^{ \pm}}{z_{23}^{ \pm} z_{24}^{ \pm}}, & +\frac{1}{3} k_{q}^{2}\left(\frac{z_{23}^{ \pm}}{z_{24}^{ \pm} z_{34}^{ \pm}}-\frac{z_{12}^{ \pm}}{z_{14}^{ \pm} z_{24}^{ \pm}}\right) \\
V_{p}^{3} \equiv k_{p}^{1} \frac{z_{14}^{ \pm}}{z_{31}^{ \pm} z_{34}^{ \pm}}+k_{p}^{2} \frac{z_{24}^{ \pm}}{z_{32}^{ \pm} z_{34}^{ \pm}}, & +\frac{1}{3} k_{q}^{3}\left(\frac{z_{31}^{ \pm}}{z_{14}^{ \pm} z_{34}^{ \pm}}-\frac{z_{23}^{ \pm}}{z_{24}^{ \pm} z_{34}^{ \pm}}\right) .
\end{array}
$$

The amplitude $\mathcal{A}_{0000}$ is independent of the position of the unintegrated vertices. The Möbius transformations are given by

$$
z_{i}^{+} \rightarrow \frac{a z_{i}^{+}+b}{c z_{i}^{+}+d}, \quad z_{i}^{-} \rightarrow \frac{a^{*} z_{i}^{-}+b^{*}}{c^{*} z_{i}^{-}+d^{*}}
$$

with $a d-b c=1$. Therefore, $z_{i j}^{ \pm}$transforms as

$$
z_{i j}^{+} \rightarrow \frac{z_{i j}^{+}}{\left(c z_{i}^{+}+d\right)\left(c z_{j}^{+}+d\right)}, \quad z_{i j}^{-} \rightarrow \frac{z_{i j}^{-}}{\left(c^{*} z_{i}^{-}+d^{*}\right)\left(c^{*} z_{j}^{-}+d^{*}\right)},
$$

naturally leading to the definition of the invariant cross-ratios

$$
x^{ \pm} \equiv \frac{z_{41}^{ \pm} z_{23}^{ \pm}}{z_{12}^{ \pm} z_{34}^{ \pm}}, \quad 1-x^{ \pm}=\frac{z_{13}^{ \pm} z_{24}^{ \pm}}{z_{12}^{ \pm} z_{34}^{ \pm}}, \quad \frac{x^{ \pm}}{1-x^{ \pm}}=\frac{z_{41}^{ \pm} z_{23}^{ \pm}}{z_{13}^{ \pm} z_{24}^{ \pm}} .
$$

In terms of $x^{ \pm}$, equation (A.38) can be expressed as

$$
I_{4}\left(s, u ; x^{ \pm}\right)=\delta^{26}\left(k^{1}+k^{2}+k^{3}+k^{4}\right)\left(\frac{x^{-}}{x^{+}}\right)^{\frac{u}{4 \mathcal{T}}}\left(\frac{1-x^{-}}{1-x^{+}}\right)^{\frac{t}{4 \mathcal{T}}}
$$

and the Möbius invariance of $\mathcal{A}_{0000}$ can be easily checked using the transformations (A.42) and

$$
d z_{4}^{+} d z_{4}^{-} \rightarrow \frac{d z_{4}^{+} d z_{4}^{-}}{\left(c z_{4}^{+}+d\right)^{2}\left(c^{*} z_{4}^{-}+d^{*}\right)^{2}}
$$


Therefore, the positions $z_{1}^{ \pm}, z_{2}^{ \pm}$and $z_{3}^{ \pm}$will be conveniently chosen to be $z_{1}^{ \pm}=0$, $z_{2}^{ \pm}=1$ and $z_{3}^{ \pm}=\infty$, such that $x^{ \pm}=z_{4}^{ \pm}$and

$$
\begin{aligned}
T_{m n p q}^{ \pm}= & -\frac{\left(k_{m}^{2} x^{ \pm}+k_{m}^{4}\right)\left(k_{n}^{1} x^{ \pm}+k_{n}^{3}\right)\left(k_{p}^{4} x^{ \pm}+k_{p}^{2}\right)\left(k_{q}^{3} x^{ \pm}+k_{q}^{1}\right)}{\left(x^{ \pm}\right)^{2}\left(1-x^{ \pm}\right)^{2}} \\
& \mp 2 \mathcal{T} \eta_{n q} \frac{\left(k_{m}^{2} x^{ \pm}+k_{m}^{4}\right)\left(k_{p}^{4} x^{ \pm}+k_{p}^{2}\right)}{\left(x^{ \pm}\right)\left(1-x^{ \pm}\right)^{2}} \pm 2 \mathcal{T} \eta_{m p} \frac{\left(k_{n}^{1} x^{ \pm}+k_{n}^{3}\right)\left(k_{q}^{3} x^{ \pm}+k_{q}^{1}\right)}{\left(x^{ \pm}\right)\left(1-x^{ \pm}\right)^{2}} \\
& \mp 2 \mathcal{T} \eta_{m q} \frac{\left(k_{n}^{1} x^{ \pm}+k_{n}^{3}\right)\left(k_{p}^{4} x^{ \pm}+k_{p}^{2}\right)}{\left(x^{ \pm}\right)^{2}\left(1-x^{ \pm}\right)} \mp 2 \mathcal{T} \eta_{n p} \frac{\left(k_{m}^{2} x^{ \pm}+k_{m}^{4}\right)\left(k_{q}^{3} x^{ \pm}+k_{q}^{1}\right)}{\left(x^{ \pm}\right)^{2}\left(1-x^{ \pm}\right)} \\
& \pm 2 \mathcal{T} \eta_{p q} \frac{\left(k_{m}^{2} x^{ \pm}+k_{m}^{4}\right)\left(k_{n}^{1} x^{ \pm}+k_{n}^{3}\right)}{x^{ \pm}\left(1-x^{ \pm}\right)} \pm 2 \mathcal{T} \eta_{m n} \frac{\left(k_{p}^{4} x^{ \pm}+k_{p}^{2}\right)\left(k_{q}^{3} x^{ \pm}+k_{q}^{1}\right)}{\left(x^{ \pm}\right)\left(1-x^{ \pm}\right)} \\
& +4 \mathcal{T}^{2} \eta_{m n} \eta_{p q}+4 \mathcal{T}^{2} \frac{\eta_{m p} \eta_{n q}}{\left(1-x^{ \pm}\right)^{2}}+4 \mathcal{T}^{2} \frac{\eta_{m q} \eta_{n p}}{\left(x^{ \pm}\right)^{2}} .
\end{aligned}
$$

Now, observe that all terms of the integrand of (A.37) can be written as

$$
I_{4}(U, T ; m, n, \bar{m}, \bar{n}) \equiv \int d^{2} z\left\{z^{m-2}(1-z)^{n-2} \bar{z}^{\bar{m}-2}(1-\bar{z})^{\bar{n}-2}\left(\frac{\bar{z}}{z}\right)^{U}\left(\frac{1-\bar{z}}{1-z}\right)^{T}\right\}
$$

where $\{m, n\}=0, \ldots, 4$, with $m+n \leq 4$ and the same for $\{\bar{m}, \bar{n}\}$. This restriction appears only in the chiral model and is a consequence of the conformal weights of the building blocks of the vertex operators. The normalized Mandelstam variables $S, T$ and $U$, with $S+T+U=0$, are just

$$
S \equiv \frac{s}{4 \mathcal{T}}, \quad T \equiv \frac{u}{4 \mathcal{T}}, \quad U \equiv \frac{u}{4 \mathcal{T}} .
$$

The chiral string character of this amplitude is very clear for the powers involving the Mandelstam variables appear as $\frac{\bar{z}}{z}$ and $\frac{1-\bar{z}}{1-z}$ instead of $|z|^{2}$ and $|1-z|^{2}$. The evaluation of $I_{4}$ will be done by adapting the known Kawai-Lewellen-Tye relations of [28] to the case above, which is slightly different due to the opposite phase contribution of the branch cuts.

Considering $z=x+i y, I_{4}$ can be seem as an analytic function in $y$ with four branch points,

$$
y= \pm i x, \pm i(1-x)
$$

The contour of integration of $y$ over the real line can be deformed to the imaginary line. To do that, it is useful to rewrite part of the integrand as

$$
\left(\frac{\bar{z}}{z}\right)^{U}=\bar{z}^{2 U} \frac{1}{\Gamma(U)} \int_{0}^{\infty} \alpha^{s-1} e^{-(Z \bar{Z}) \alpha} d \alpha .
$$

For a fixed $\alpha$, the exponential acts as a convergence factor in the integration and the contour deformation is well defined. The overall result is similar to a Wick-rotation. After defining $\xi \equiv x+i y$ and $\eta \equiv x-i y, I_{4}$ can be written as

$$
I_{4}=\int_{-\infty}^{+\infty} d \xi \int_{-\infty}^{+\infty} d \eta\left\{|\xi|^{m-2-U}|1-\xi|^{n-2-T}|\eta|^{\bar{m}-2+U}|1-\eta|^{\bar{n}-2+T}\right\} \times f(\xi, \eta),
$$


where $f(\xi, \eta)$ is a phase factor determined by the domain analysis of the integrals. Following closely the steps in [28], it is possible to show that

$$
I_{4}=\sin (\pi T) \times \int_{0}^{1} d \xi\left\{\xi^{m-2-U}(1-\xi)^{n-2-T}\right\} \times \int_{1}^{\infty} d \eta\left\{\eta^{\bar{m}-2+U}(\eta-1)^{\bar{n}-2+T}\right\}
$$

Now, using Euler's reflection formula,

$$
\sin \pi a=\frac{\pi}{\Gamma(1-a) \Gamma(a)}
$$

where $\Gamma(a)$ is the gamma function, and recalling that

$$
\int_{0}^{1} d x x^{a-1}(1-x)^{b-1}=\frac{\Gamma(a) \Gamma(b)}{\Gamma(a+b)},
$$

$I_{4}$ is finally written (up to a sign) as

$$
I_{4}(U, T ; m, n, \bar{m}, \bar{n})=\pi \frac{\Gamma(3-\bar{m}-\bar{n}+S)}{\Gamma(m+n-2+S)} \frac{\Gamma(m-1-U)}{\Gamma(2-\bar{m}-U)} \frac{\Gamma(n-1-T)}{\Gamma(2-\bar{n}-T)} .
$$

Given that $m, n, \bar{m}, \bar{n} \geq 0$ with $m+n \leq 4$ and $\bar{m}+\bar{n} \leq 4$, it is straightforward to see that the poles of $I_{4}$ are located at $S, T, U=0, \pm 1$ ( or $s, t, u=0, \pm 4 \mathcal{T}$ ), i.e. the expected poles associated to the physical spectrum of the model. Note, for example, that

$$
\frac{\Gamma(m-1-U)}{\Gamma(2-\bar{m}-U)}= \begin{cases}\prod_{i=1}^{m+\bar{m}-3}(m-1-U-i) & m+\bar{m} \geq 3 \\ \prod_{i=1}^{3-m-\bar{m}}(2-\bar{m}-U-i)^{-1} & m+\bar{m}<3\end{cases}
$$

and the poles occur in the $U$ channel only when $m+\bar{m}<3$. In the $T$ channel, the poles appear when $n+\bar{n}<3$. For the $S$ channel the poles occur when $m+n+\bar{m}+\bar{n}>5$ :

$$
\frac{\Gamma(3-\bar{m}-\bar{n}+S)}{\Gamma(m+n-2+S)}= \begin{cases}\prod_{i=1}^{5-m-n-\bar{m}-\bar{n}}(3-\bar{m}-\bar{n}+S-i) & m+n+\bar{m}+\bar{n} \leq 5 \\ \prod_{i=1}^{m+n+\bar{m}+\bar{n}-5}(m+n-2+S-i)^{-1} & m+n+\bar{m}+\bar{n}>5\end{cases}
$$

The cancellation of the other poles of the gamma functions works exactly as described in $[12]$.

Open Access. This article is distributed under the terms of the Creative Commons Attribution License (CC-BY 4.0), which permits any use, distribution and reproduction in any medium, provided the original author(s) and source are credited. 


\section{References}

[1] L. Mason and D. Skinner, Ambitwistor strings and the scattering equations, JHEP 07 (2014) 048 [arXiv: 1311.2564] [INSPIRE].

[2] F. Cachazo, S. He and E.Y. Yuan, Scattering of Massless Particles in Arbitrary Dimensions, Phys. Rev. Lett. 113 (2014) 171601 [arXiv:1307.2199] [INSPIRE].

[3] O. Hohm, W. Siegel and B. Zwiebach, Doubled $\alpha^{\prime}$-geometry, JHEP 02 (2014) 065 [arXiv:1306.2970] [INSPIRE].

[4] I. Bandos, Twistor/ambitwistor strings and null-superstrings in spacetime of $D=4,10$ and 11 dimensions, JHEP 09 (2014) 086 [arXiv: 1404.1299] [INSPIRE].

[5] W. Siegel, Amplitudes for left-handed strings, arXiv:1512.02569 [INSPIRE].

[6] J. Gamboa, C. Ramirez and M. Ruiz-Altaba, Null spinning strings, Nucl. Phys. B 338 (1990) 143 [INSPIRE].

[7] J. Gamboa, C. Ramirez and M. Ruiz-Altaba, Quantum null (super)strings, Phys. Lett. B 225 (1989) 335 [INSPIRE].

[8] E. Casali and P. Tourkine, On the null origin of the ambitwistor string, JHEP 11 (2016) 036 [arXiv: 1606. 05636] [INSPIRE].

[9] N. Berkovits, Infinite Tension Limit of the Pure Spinor Superstring, JHEP 03 (2014) 017 [arXiv: 1311.4156] [INSPIRE].

[10] E. Casali and P. Tourkine, Windings of twisted strings, Phys. Rev. D 97 (2018) 061902 [arXiv: 1710.01241] [INSPIRE].

[11] N. Berkovits and M. Lize, Field theory actions for ambitwistor string and superstring, JHEP 09 (2018) 097 [arXiv: 1807.07661] [INSPIRE].

[12] Y.-t. Huang, W. Siegel and E.Y. Yuan, Factorization of Chiral String Amplitudes, JHEP 09 (2016) 101 [arXiv : 1603. 02588] [INSPIRE].

[13] T. Azevedo, R.L. Jusinskas and M. Lize, Bosonic sectorized strings and the $(D F)^{2}$ theory, arXiv:1908.11371 [INSPIRE].

[14] T. Adamo, E. Casali and D. Skinner, Ambitwistor strings and the scattering equations at one loop, JHEP 04 (2014) 104 [arXiv: 1312.3828] [INSPIRE].

[15] Y. Geyer and R. Monteiro, Two-Loop Scattering Amplitudes from Ambitwistor Strings: from Genus Two to the Nodal Riemann Sphere, JHEP 11 (2018) 008 [arXiv:1805.05344] [INSPIRE].

[16] M. Yu, C. Zhang and Y.-Z. Zhang, One loop amplitude from null string, JHEP 06 (2017) 051 [arXiv: 1704.01290] [INSPIRE].

[17] K. Lee, S.-J. Rey and J.A. Rosabal, A string theory which isn't about strings, JHEP 11 (2017) 172 [arXiv: 1708.05707] [INSPIRE].

[18] R.L. Jusinskas, Notes on the ambitwistor pure spinor string, JHEP 05 (2016) 116 [arXiv: 1604.02915] [INSPIRE].

[19] R.L. Jusinskas, Towards the underlying gauge theory of the pure spinor superstring, JHEP 10 (2019) 063 [arXiv: 1903.10753] [INSPIRE].

[20] K. Ohmori, Worldsheet Geometries of Ambitwistor String, JHEP 06 (2015) 075 [arXiv: 1504.02675] [INSPIRE]. 
[21] S. Mizera, Aspects of Scattering Amplitudes and Moduli Space Localization, Ph.D. Thesis, Perimeter Inst. Theor. Phys. (2019) [arXiv:1906.02099] [INSPIRE].

[22] S. Mizera, Scattering Amplitudes from Intersection Theory, Phys. Rev. Lett. 120 (2018) 141602 [arXiv: 1711.00469] [INSPIRE].

[23] E. Casali, Y. Herfray and P. Tourkine, The complex null string, Galilean conformal algebra and scattering equations, JHEP 10 (2017) 164 [arXiv:1707.09900] [INSPIRE].

[24] R.A. Reid-Edwards, Ambitwistor String Theory in the Operator Formalism, JHEP 06 (2016) 084 [arXiv: 1511.08406] [INSPIRE].

[25] R.A. Reid-Edwards and D.A. Riccombeni, A Superstring Field Theory for Supergravity, JHEP 09 (2017) 103 [arXiv: 1701.05495] [INSPIRE].

[26] S.G. Naculich, Scattering equations and BCJ relations for gauge and gravitational amplitudes with massive scalar particles, JHEP 09 (2014) 029 [arXiv:1407.7836] [INSPIRE].

[27] M.M. Leite and W. Siegel, Chiral Closed strings: Four massless states scattering amplitude, JHEP 01 (2017) 057 [arXiv: 1610.02052] [INSPIRE].

[28] H. Kawai, D.C. Lewellen and S.H.H. Tye, A Relation Between Tree Amplitudes of Closed and Open Strings, Nucl. Phys. B 269 (1986) 1 [INSPIRE]. 